\title{
K-Means Clustering - An Access to Overcome Uncertainty in Data Clustering of Distributed Networks
}

\author{
P.V.Ashwathy Devraj, S.Manju, D.Pavithra, M.Nithya
}

\begin{abstract}
Clusteringisaprocedureofdividing a lot of information (or objects) into a lot of significant sub-classes, called bunches, help clients comprehend the characteristic gathering or structure in an informational index. Clustering has wide applications, in Economic Science (particularly statistical surveying), Document order, Recognition, Spatial Data Analysis and Image Processing Thewaytowardgatheringalotofphysicalor dynamic items into classes of same articlesis called grouping. A group is an accumulation of information questions that are near each other inside a similar bunch and are not atall like the articles in different groups. A bunch of information articles can be dealt with together as one gathering thus might be considered as a type of informationpressure. In spite of the fact that characterization is a compelling methods for recognizing gatherings or classes of items, it requires the frequently exorbitant naming of a huge arrangement of preparing tuples or examples, which the classifier uses to display each gathering.

Clustering is likewise called information division in certain applications since clustering parcels substantial informational collections into gatherings as per their similitude. A decent clustering strategy will deliver top notch groups intra- class (that is, intra-group) comparability is high, the between class likeness is low and nature of a clustering result additionally relies upon closeness measure utilized by the technique and, the nature of a clustering technique is likewise estimated by its capacity to find a few or the majority of the concealed examples, nonetheless, target assessment is dangerously normally done by human/master examination. When all is said in done, the significant grouping techniques can be isolated into the accompanying classifications Partitioning strategies, various leveled techniques, Density-based strategies, Grid-based strategies, Model- basedtechniques.Thispaperutilizesfuzzycimplies grouping with PSO for clustering procedure of diabetic forecastdataset.
\end{abstract}

\section{INTRODUCTION}

Human body needs vitality for initiation. The starches are isolated down to glucose, which is the vital vitality hotspotfor human body cells. Insulin is expected to transport the glucose into human body cells. The blood glucose is provided with insulin and glucagon hormones created by pancreas. Insulin hormones delivered with the assistance of beta cells of the islets of Langerhans and glucagon hormones are created by the alpha cells of the islets of

Revised Manuscript Received on September 10, 2019.

Ms.P.V.Ashwathy Devraj, Assistant Professor, Department of Computer Science and Engineering, Karpagam College of Engineering, Coimbatore, Tamilnadu, India.

(E-mail: ashnov22@gmail.com)

S.Manju, Student, Department of Computer Science and Engineering, Karpagam College of Engineering, Coimbatore, Tamilnadu, India.

D.Pavithra, Student, Department of Computer Science and Engineering, Karpagam College of Engineering, Coimbatore, Tamilnadu, India.

M.Nithya, Student, Department of Computer Science and Engineering, Karpagam College of Engineering, Coimbatore, Tamilnadu, India.
Langerhans in the pancreas. At the point when the blood glucose expands, beta cells are animated and insulin is given to the blood. Insulin empowers blood glucose to get into the body cells and this glucose is utilized for vitality. So blood glucose is kept in a little range. Diabetes is the perpetual contamination with the opportunity to purpose a normal human offerings emergency. As consistent with International Diabetes Federation 382 a first-rate many individuals are residing with diabetes over the complete world.By 2035, this will be twice as 592 million. In any case, early forecast of diabetes is all the more testing undertaking for therapeutic experts because of complex association on different variables.

Diabetes influences human organs, for instance, kidney, eye, coronary heart, nerves, foot and so forth.In this paper we utilize fuzzy c-implies with PSO for grouping process. Clustering investigation is a nonexclusive term for factual systems intended to distinguish gatherings of perceptions that have comparable characteristics, and it is an essential part of unsupervised example acknowledgment. It can assist the client with distinguishing the structure of information and rearrange the unpredictability of information from mass data. The client can comprehend the suggested data behind by separating this information. Clustering is thewaytowardgatheringinformationobjects

intoalotofdisjointclasses,soprotestsinside a class have high similitude to one another, while questions in independent classes are increasingly disparate.

As an unsupervised characterization strategy, clustering examination have been connected to awide scope of issues, including exploratory example acknowledgment, information investigation, information mining, picture division, PC vision, fuzzy control and numerical programming . In clustering calculation dependent on target work, the fuzzy c-implies calculation hypothesis is the most total one and has been utilized broadly. Tragically, the aftereffects of fuzzyc-implies procedure rely upon the clustering focus instatement in light of the fact that their hunt depends on slope climbing strategies to conquer a portion of the weaknesses offuzzy c-implies, there has been much examination on the utilization of developmental calculation to parcel information into fuzzy grouping. K.S.Asultan and S.Selim proposed the Simulated Annealing Algorithm (SA) to defeat a portion of these breaking points and detailed promising outcomes in 1993. In 1994, G.P.Bab and Mu.Murty utilized the Evolution Strategies (ES) to 
parcel information into fuzzy clustering.Particle Swarm Optimization (PSO) has been proposed in 1995. PSO is a generally ongoing heuristic motivated by the movement of a winged creature rush which has been observed to be very effective in a wide assortment of improvement errands. Its rapid of union and its relative effortlessness make PSO an exceptionallyreasonable

competitorutilizedforsettlingwithasolitary target work. In this paper, we give a FCM approach guided by PSO which is characterized asPSOFCM.

\section{LITERATURE SURVEY}

\section{Utilization of the Data Mining Methods in Diabetes Prediction:}

Information science techniques can possibly profit another logical field by revealing new insight into normal inquiries. One errand is help to make expectations on therapeutic information. Diabetes mellitus or fundamental diabetes is a sickness caused because of the expansion dimension ofblood glucose. Different conventional techniques, inlightofthephysicalandsynthetictests, are accessible for diagnosing diabetes. The strategies dependent on the information mining methods can be successfully connected for the hypertension chance expectation. In this paper, we investigate the early expectation of the diabetes by meansof five distinct information mining strategies including: GMM, SVM, Logistic relapse, ELM,ANN.

\section{Data Mining in Healthcare for Diabetes Mellitus:}

Illness finding is one of the applications where the information mining devices are demonstrating victories. Diabetes infectionis the main source of the demise everywhere throughouttheworldinthepreviousyears.A few analysts are utilizing measurable information. The accessibility of a lot of medicinal information prompts the requirement for incredible mining devices to enable wellbeing to mind experts in the determination of diabetes sickness. Utilizing information mining system in the determination of diabetes infection has exhaustively researched, appearing satisfactory dimensions of exactness. As of late scientists are exploring the impact of hybridizing more than one system indicating upgradedresultsintheconclusionofdiabetes infection. Nonetheless, utilizing information mining systems to recognize a reasonable treatment for the diabetes illness patients has gotten less consideration.

This paper used to distinguishes holes in the exploration on diabetes sickness determination and treatment. It helps methodically close those holes to find if applying information mining procedures to diabetes sickness treatment information can give as solid execution as that accomplished in controlling and diagnosing diabetes infection.

Use of k-Means Clustering calculation for the forecast of Students' Academic Performance:

Thecapacitytoscreentheadvancement of the understudies' scholastic execution is a basic issue to the scholarly network ofhigher learning. A framework for investigating understudies' outcomes dependent on group examination and employments of standard measurable calculations to mastermind their scores information as per the dimension of their execution is portrayed. In this paper,we are likewise actualized k-mean clustering calculation for investigating understudies' outcome information. The model was joined with the deterministic model to break down the understudies' aftereffects of the private Institution in Nigeria which is a decent benchmark to screen the movement of scholarly execution of understudies inhigher Institutions to make a successful choice by the scholasticorganizers.

\section{Information Mining K-Clustering Problem:}

In the measurement and information mining, $\mathrm{k}-$ impliesgroupingisoutstandingfor its proficiency in clustering substantial informational indexes. The point is to gather information focuses into the groups with the end goal that comparable thingsare lumped together in a similar bunch. In like manner, given a lot of articles together with their qualities, theobjectiveistoseparatetheitems into $\mathrm{k}$ bunches to such an extent that objects lying in one group ought to be as close as conceivable to one another's (homogeneity) and articles lying in various bunches are further separated from one another.

In any case, there exist a few imperfections in the established K-implies clustering calculation. As indicated by the strategy, first calculation is delicate to choosing starting Centroid and can be effectively caught at a neighborhood leastwithrespecttotheestimation(thewhole of squared mistakes) utilized in the model. Also, then again, the Kimplies issue in the terms of finding a worldwide negligible entirety of the squared mistakes is NP-hard notwithstanding when the quantity of the bunch is equivalent 2 or the quantity of quality for the information point is 2 , so finding the ideal grouping is accepted to be computationally obstinate.

\section{Information Mining Clustering Techniques:}

A reviewwith the approach increment in medical problems in our everyday life, information mining has been a basic part to bring the learning and to shape diverse examples. Exactness is extremely fundamental once it includes in thepatient consideration and mechanizing this has substantial amount of information upgrades the standard of the total framework. Information mining chips away at the main of KDD (Knowledge Discovery in Databases) calculations. Information mining devices analyze the accompanying side effects, causes, medicines and negative impacts in ordertocontinuetoexplorewhatactivitycan be demonstrated most straightforward for a gathering of patients. The information from the guide associations are voluminous and heterogeneous. It must be gathered and kept inthesortedoutsortandtheirjoiningpermits the development join medicinal framework. Information mining in wellbeing offerssome boundless conceivable outcomes for dissecting models less obvious or coveredup tobasicinvestigationstrategies.Inthispaper, the different procedures are analyzed on which examine methods have been connected. 


\section{EXISTING SYSTEM \& RESULTS}

Questionable information grouping has been perceived as basic undertaking in the examination of information mining. Such huge numbers of unified clustering calculations are stretched out by characterizing new separation or comparability estimations to handlethis issue. With the quick improvement of the system applications, these incorporated strategies demonstrate their impediments in directing information grouping in a huge powerful conveyed shared system becauseof the protection and security concerns or the specialized limitations raised by the distributive conditions. In this paper, we going to propose a novel dispersed dubious information clustering calculation, in which the concentrated worldwide grouping arrangement approximated by performing disseminated clustering.

To abbreviate the execution time, the decrease strategy is connected to change the proposed technique into its deterministic structure bysupplanting each dubious information object with its normal centroid. At long last, the quality weight-entropy gularization strategy upgrades the proposed conveyed grouping technique to accomplish better outcomes in information clustering and separate the basic highlights for bunch recognizable proof. The investigations on both manufactured just as genuine information have demonstrated the productivityandprevalenceoftheintroduced calculation.

\section{PROPOSED SYSTEM}

Clustering is additionally known as information division in certain applications since grouping parcels expansive informational indexes into gatherings as per their likeness. A decent grouping strategy willdeliverfantasticbunchesintraclass(that is, intra-group) comparability is high, the between class closeness is low the nature of a clustering result likewise relies uponlikeness measure utilized by the technique and the nature of a grouping technique is additionally estimated by its capacity to find a few or the majorityof the shrouded examples, be that as it may, target assessment is the dangerous. it normally done by human/master assessment. As a rule, the major clustering techniquesare grouped into the accompanying classes Partitioning strategies, Hierarchical techniques, Densitybased techniques, Grid- based strategies, Model-based techniques. . This paper utilizes fuzzy c-implies grouping with PSO for clustering procedure of diabetic expectation data set.

\section{ALGORITHM}

Step1: Data is collected based on diabetic disease

Step2: collected data is given as input to the MATLAB

Step 3: preprocessing of the data will be done. Preprocessing is nothing but conversation of data elements in to numeric values

Step4: clustering using k-means

Step 5: applying PSO for efficient search of members of the group in preprocessed data

Step 6: fuzzy c-means is applied along with the Particle Swarm Optimization

Step 7: Clustering results
Step8: comparison of k-means and fuzzy c-means with PSO

\section{BLOCKDIAGRAM}


\section{METHODOLOGY}

Fuzzy with PSO:

The Fuzzy C-implies calculation is the more appropriate partitional grouping approach for taking care of huge dataset concerning execution time. This paper presents another Modified Particle Swarm Optimization strategy that consolidates the best highlights of PSO and fuzzy C-implies calculations for effective clustering.

Fuzzy C-implies strategy is considered as an able parceling calculation for clustering a huge information vectors into an all-around accepted and determined number of groups. A clustering strategy Fuzzy C-Mean (FCM) is a superior performing groupingcalculation when contrasted with K-mean as bunch limits are not any more hard limits,yetit is like wise reliant after clustering focus instatement. We can get better starting grouping centroids utilizing for some different systems and along these lines it might lead Fuzzy C- implies calculation to perform better

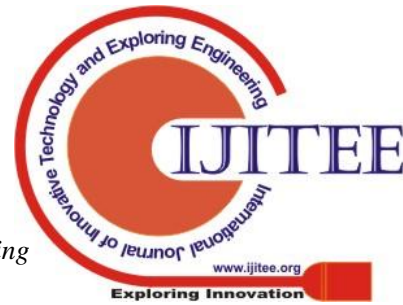


during the time spent finding the most ideal clustering focuses by the method for improving the clustering centroid. The Particle Swarm Optimization (PSO) calculation is the heuristic system that enhances an issue by iteratively endeavoring to improve a competitor arrangement as to a given proportion of value. The better beginning group centroid can be gotten by utilizing PSO. We propose PSO joined with Fuzzy C-implies calculation that creates a snappy and better clustering and furthermore it endeavors to stay away from in getting interested towards a restricted most ideal result.

\section{CONCLUSION}

Our task depends on grouping method. This fuzzy cimplies alongside PSO is utilized on diabetic dataset. $\mathrm{K}$ implies clustering process is additionally utilized on diabetic dataset. At that point correlation is made for both clustering process. From the examination the outcomes demonstrate that grouping done utilizing fuzzy methods with PSO is compelling than $\mathrm{k}$ implies. The precision chart is likewise made for both grouping process.

\section{REFERENCES}

1. Devi, M. Renuka, and J. MariaShyla. "Analysis of Various Data Mining Techniques to Predict Diabetes Mellitus." International Journal of Applied Engineering Research 11.1 (2016):727-730.

2. Berry, Michael 1., and Gordon Linoff. Data mining techniques: for marketing, sales, and customer support. John Wiley \& Sons, Inc., 1997

3. Witten, Ian H., et al. Data Mining: Practical machine learning tools and techniques. Morgan Kaufmann,2016.

4. Emoto, Takuo, et al. "Characterization of gut microbiota profiles in coronary artery disease patients using data mining analysisof terminal restriction fragment length polymorphism: gut microbiota could be a diagnostic marker of coronary artery disease." Heart and vessels 32.1 (2017):39-46.

5. Giri, Donna, et al. "Automated diagnosis of coronary artery disease affected patients using LDA, PCA, ICA and discrete wavelet transform." Knowledge-Based Systems 37 (2013):274-282.

6. Fatima,Meherwar,andMarufPasha. "Survey of Machine Learning Algorithms for Disease Diagnostic."Journal of Intelligent Learning Systems and Applications 9.01 (2017):1.

7. Huang,Guang-Bin,Qin-YuZhu, and Chee-KheongSiew. "Extreme learning machine: theory and applications." Neurocomputing 70.1 (2006):489-501.

8. Nagaraj, B., and R. Murugananth. "Optimum tuning algorithms for PID controller-a soft computing approach." IPPTA Journal 22, no. 2 (2010): 127-130..

9. Tiwari, Mukesh, Jan Adamowski, and KazimierzAdamowski."Water demand forecasting using extreme learning machinesof Water and Land Development 28.1 (2016): 37-52.

10. A new facial expressionrecognition based on curvelet transform and online sequential extreme learning machine initialized with spherical clustering." Neural Computing and Applications 27.1 (2016): 131-142 